\title{
Age ANd Human Lenticular Fluorescence
}

\author{
R. A. Weale \\ King's College London, Age Concern Institute of Gerontology, Cornwall House, Waterloo Rd., \\ London SE1 8WA, and University College Hospital, Eye Department, Gower St., London WC1, United \\ Kingdom \\ (Paper JBO/LSF-03 received Mar. 8, 1996; revised manuscript received Apr. 24, 1996; accepted for publication Apr. 30, \\ 1996)
}

\begin{abstract}
This critical survey of studies on the fluorescence of human eye lenses covers basic problems of metrology and their relation to comparative measurements on extracts, on in vivo, and on in vitro material. Attention is drawn to the effects of lenticular refraction and absorbance, and to potential photic involvement on the part of ambient illumination. The extent to which fluorescence may interfere with vision is also reviewed. () 1996 Society of Photo-Optical Instrumentation Engineers.
\end{abstract}

Keywords light scatter and fluorescence; lenticular fluorescence; lenticular absorbance; ocular aging; age; haze.

\section{VALUE OF LENTICULAR FLUORESCENCE STUDIES}

Lenticular fluorescence has been known for the last 150 years or so, and, though important qualitative observations were reported earlier in the twentieth century, ${ }^{1}$ quantitative analysis began only after the Second World War, ${ }^{2}$ probably following the development of photoelectric devices.

Vogt drew attention to the changes in the coloration of lenticular fluorescence which correlate with age, but did not distinguish between forward and backward fluorescence. ${ }^{3}$ Qualitative studies evidently could not distinguish between effects due to multiple fluorphors on the one hand and complications due to pigment absorption on the other. $\mathrm{Klang}^{2}$ was able to demonstrate the presence of at least two different fluorophors and thereby established the analytic value of fluorescence studies.

Interest in lenticular fluorescence further developed with the realization that it increases with age, and also following the discovery that it is distributed nonuniformly in the lenticular matrix. ${ }^{4}$

Information in vivo was not obtained till the last decade or two. This is probably due to the increase in the sensitivity of radiometric methods, but Klang had demonstrated the feasibility of in vivo measurements when photoelectric techniques were being developed, and visual photometry might have been available had the interest been there.

Consequently in vivo lenticular fluorescence has only recently become a tool suitable for comparative measurements within and between populations, and its value, especially in connection with diabetic lens problems, has only lately been recognized without being fully exploited even now.

\subsection{METROLOGY}

Since the measurement of fluorescence entails the use of an exciting beam with a short-wavelength radiation and the detection of emitted radiation invariably with a longer wavelength, the separation of the two is important. Except when forward fluorescence is studied, the separation can be achieved by the physical segregation of the two beams, as is true in in vivo when a careful distinction between the two directions has to be ensured. It is supplemented optically. Because of the relatively low intensity of the emitted light, spectral filters rather than the preferable monochromatic radiations have to be employed to provide an adequate signal-tonoise ratio. As they have finite bandwidths, overlap between them needs firm control. Only a few workers in the field (see Ref. 5) have measured the extended absorbances of the filters they have used in order to assure themselves of the absence of spectral "feet" which treacherously transmit in distant spectral regions, potentially vitiating their results.

In vivo measurements suffer from a further uncertainty that is frequently overlooked. Although the exciting beam is usually defined with respect to its point of entry and the pupillary center, and, like the emitted beam, regarding the angle it includes with the visual axis, the point at which emission takes place is a matter of unconfirmed surmise. There are several reasons for this. The depth of the anterior chamber is not recorded; it decreases systematically with age. Consequently the point of entry of the exciting beam into the lens becomes indeterminate, with the result that a detector of the radiation emitted over a wide volume can offer no certainty about the locus of provenance. It has to be said that this

1083-3668/96/\$6.00 (C) 1996 SPIE 
result of the gradient of the lenticular refractive index ${ }^{6}$ is not peculiar to fluorescence measurements; it introduces a great deal of uncertainty also into conclusions drawn from topographic studies of the lens, for example, by means of Scheimpflug photography.

Detailed spectral analyses of the emitted light are rare (see Refs. 7 and 8). There are two attendant problems, one of which is partly related to the difficulty addressed above. Lenticular coloration affects the spectral distribution of the emitted light in two ways. First, the exciting light is absorbed and causes fluorescence, depending on the absorbance of the relevant fluorophor; and second the emitted light is absorbed en route to the lens surface from which it emerges again, depending on the absorbance of any pigment it traverses. Hence the collected emitted light in all probability consists of a mix of radiations from different fluorophors, located in different parts of the lenticular interior. This raises important points.

No detailed action spectrum appears to have been published for human lens fluorescence, even though this can be attempted in principle. To be specific, what is required, for different age groups, is the spectral distribution of exciting radiations, based on an equal-quantum spectrum, and eliciting a constant emission spectrum. Here constancy refers both to amplitude and spectral distribution. While this would not of itself overcome the difficulty of the location of one or more fluorophors, it would assist in the definition of their spectral efficacy, and help to relate them to known chromophores. It is likely that this approach would signally refine the existing data, for example, on the variation of fluorescence as a function both of age and of the duration of diabetes.

\subsection{AGE}

In industrialized countries with obligatory registrations of birth, there is no metrological problem attached to age that has not been mentioned in the previous section. The interpretation of measurements could be refined by the application of correction factors, if not, indeed, ad hoc measurements, of such variables as the depth of the anterior chamber, lens thickness, and lenticular coloration, which are all age dependent. At present, studies indicate a great deal of variability (see Figure 1), and it remains to be seen whether refinements in measuring techniques justify the application of allowances for the above variables.

\subsection{DISEASE}

This applies also to in vivo studies of lens color in pathological conditions, such as diabetic and other cataracts. However, the simple "one-spectral-band" measurement used in all population studies so far needs replacing by a method involving at least two spectral measuring bands. The reason is that the

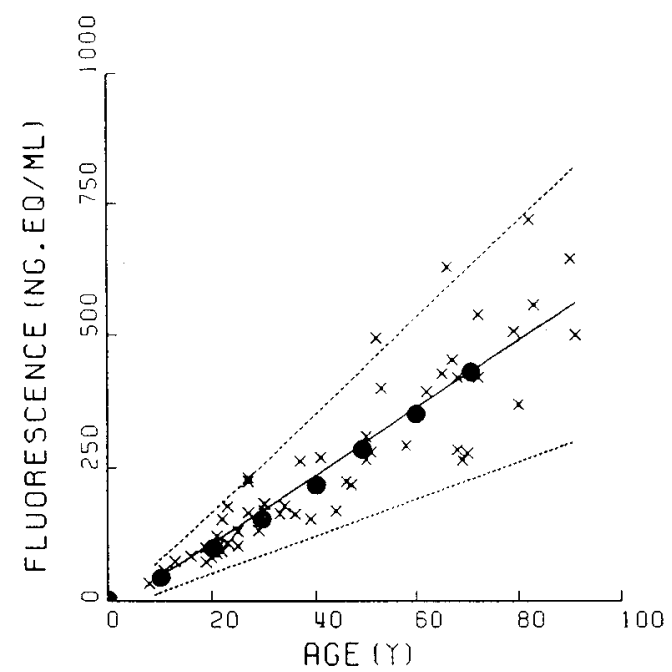

Fig. 1 Peak fluorescence (cf. Fig. 8) as a function of age of healthy subjects. Solid line, regression; dotted lines, 95\% confidence limits; filled circles, values predicted on the basis of absorbance and a variable conversion efficiency. (After Refs. 3 and 29.)

absorbance of the pigment accumulating in the lens as a result, for example, of diabetes, is distinguishable from that of other pigments accumulating merely due to aging processes; the use of more than one spectral band would render this difference quantifiable and also provide a specific measure for the abnormal fluorescence.

\subsection{COMPOSITION}

The chemical analysis of fluorescent compounds derived from the human lens is carried out on solutions (see later discussion) and does not present any significant radiometric problem. Van den Berg ${ }^{9}$ has measured the quantal conversion efficiency in vivo, and there is little doubt that, once they are obtained, in vitro values are likely to be appreciably greater. The reason is that the configurations of molecules in situ tend to differ from those in solution: in the latter instance they tend to unravel, with more photosensitive sites becoming exposed., 80 The result is that a fixed exciting flux is liable to convert to a larger quantal output than in in situ.

\subsection{RELATIVE ADVANTAGES OF DIFFERENT TYPES OF MEASUREMENT ON LENSES}

The disadvantages of studying intact lenses, whether in situ or not, have been spelled out with special reference to the uncertainty of the loci of photoconversion and also regarding spectral analysis of the emitted radiation. In young lenses it makes little difference whether axial measurements are done forward or backward, ${ }^{3}$ but it can be shown that this no longer holds for older lenses. For example, whereas age leads to a redward shift of the emergent fluorescent light in the forward case, it is blueward in the backward variety. 


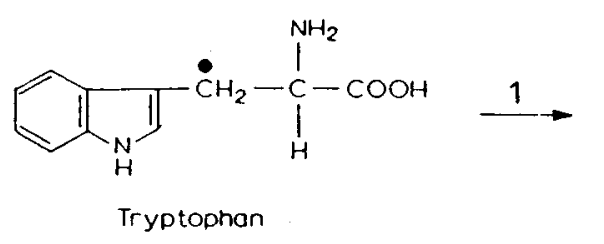

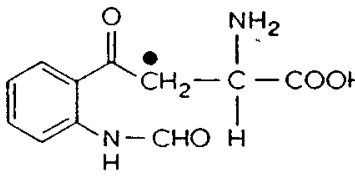

$\imath-N^{\prime}-$ Formylkynurenine<smiles>CC(CCC(=O)c1ccccc1N)C(=O)O</smiles>

Kynurenine
(F2)

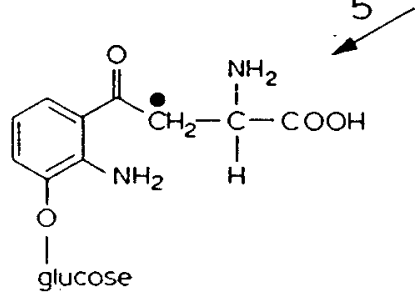

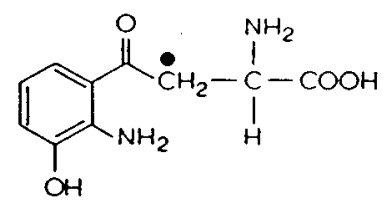

3-Hydraxykynurenine

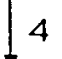<smiles>C[C@H](N)C(=O)O</smiles>

3-Hydroxyanthranilic acid + Alonine

Fig. 2 Metabolism of $\left[{ }^{14} \mathrm{C}\right]$ tryptophan (labeled carbon atom marked with dot): $\mathrm{F} 2$ is the $0-\beta$-D-glucoside of 3-hydroxy-L-kynurenine. (Reproduced courtesy of R. Van Heyningen and Ciba Foundation.)

Access to the nucleus is achieved at present only by section, although Pierscionek's ${ }^{11}$ keyhole approach could prove very valuable, for example, in demonstrating whether the highly transient fluorescent complex arising from flash illumination of the lens surface ${ }^{12}$ is detectable also in the nucleus.

\section{MeAsurements ON FLUOROPHORES}

It has been known for a long time that fluorescence can be excited in the lens in more than one spectral region, and that more than one fluorophor is likely to be involved. Generally one distinguishes between tryptophan and nontryptophan fluorescence. Tryptophan, an aromatic amino acid, absorbs maximally at $\sim 270 \mathrm{~nm}$ and fluoresces between 332 and $342 \mathrm{~nm}$ when bound to protein, but at 350 to 360 $\mathrm{nm}$ when free. ${ }^{13}$ Since the cornea absorbs $50 \%$ of the incident radiation at $320 \mathrm{~nm}$, the lenticular conversion of natural energy even by bound tryptophan is not going to be negligible. However, the lens also contains fluorescent material in specific proteins, excited at $360 \mathrm{~nm}$ and emitting in the range of 420 to $440 \mathrm{~nm}$. A compound dialyzed mainly from younger human lenses and fluorescing with blue light ${ }^{14}$ probably belongs to this group (but see later discussion). Other compounds absorbing in the vio- let part of the spectrum and fluorescing at $\sim 500 \mathrm{~nm}$ are likely to have a more powerful adverse visual effect.

Some fluorescent compounds, identified in human lens extracts and showing significant variations with age, have low molecular weights. They include L-kynurenine, the $O-\beta$-D-glucoside of 3-hydroxy-L-kynurenine, and one related to the latter, but with the $\alpha$-amino group protected or absent. ${ }^{15}$ Their compositions are shown in Figure 2. Kynurenine is derived from tryptophan and the glucoside from both tryptophan and glucose, and physiological concentrations of the compounds can act as photosensitizers (see Ref. 16). Another substance with an absorption spectrum similar to the glucoside appears to be so photosensitive as to be extractable best in the dark: ${ }^{17}$ no attempt seems to have been made by a noninvasive method to detect its presence in the living human eye, for example, after a night's sleep. However, Lerman et al. ${ }^{18}$ were able to induce blue fluorescence in a 3-day-old lens following UV irradiation even though no fluorescence could be detected in the lens of a newborn baby. If confirmed, this observation could mean that photosensitization is effected by the lens being exposed to some part of the (solar) spectrum. 

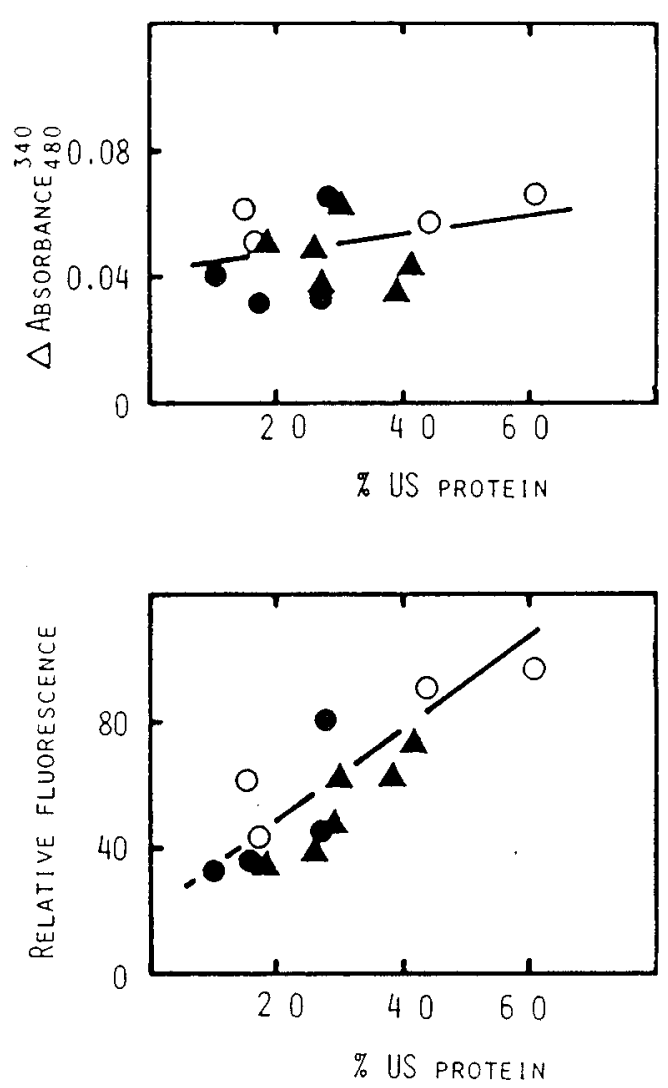

Fig. 3 Ordinate: (top) absorbance; (bottom) relative fluorescence; abscissa: percentage of urea-soluble protein. (After Ref. 20.)

The spectral fluorescent band is not characteristic of any one group of compounds. For example, we noted above that a soluble compound, found mainly in young lenses, fluoresces with blue light. But this also applies to some insoluble proteins, the concentration and degree of fluorescence of which systematically increase with age. This is true of both normal and cataractous lenses. ${ }^{19,20}$

The latter authors illuminated "cryostat-thin" sections of lenses with a band of 300 to $400 \mathrm{~nm}$, and distinguished the cortex from the nucleus in material that had not been improved as a result of being kept for about 1 month at $-20^{\circ} \mathrm{C}$. Extracts were separated into water-soluble (ws), urea-soluble (us), and urea-insoluble (ui) moieties. Although a cortex would occasionally fluoresce blue, fluorescence of aged normal and cataractous lenses was confined to the colored nucleus. The relative fluorescence of whole normal lenses, and the cortices of cataractous ones, increased linearly with the fraction of us protein (Figure 3). It is not obvious why the cortices of lenses with nuclear cataracts should behave in such a manner, but slicing the material may involve contamination, and no control of this has been reported.

This comment is underlined by the observation that, in intact lenses, the increase in fluorescence observed during the first four decades (Figure 4)

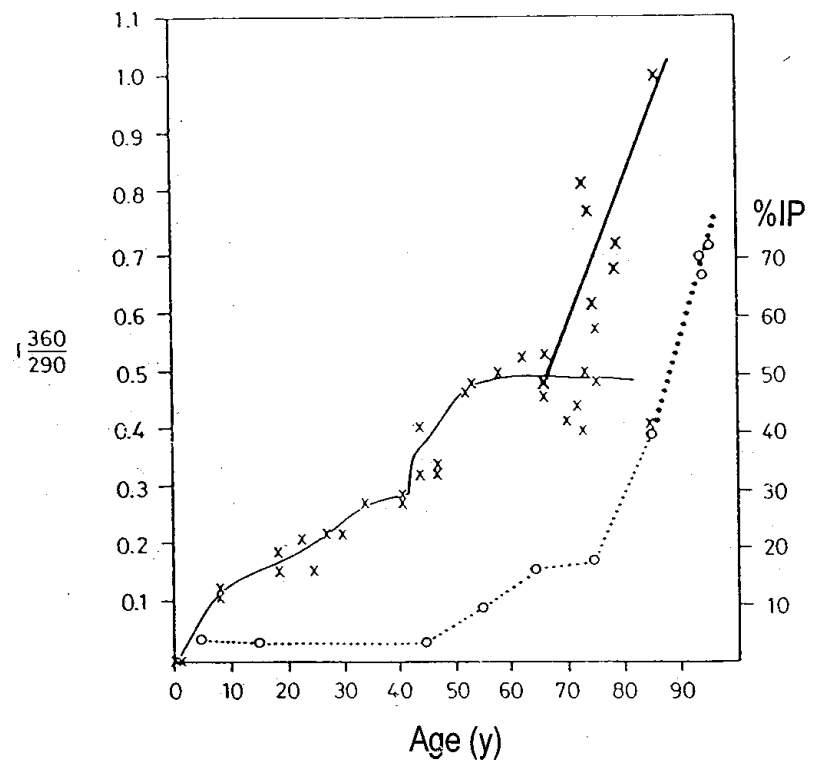

Fig. 4 Left ordinate: ratio of fluorescence intensity emitted at 420 to $440 \mathrm{~nm}$ (excited by $360 \mathrm{~nm}$ ) to fluorescence intensity emitted at $332 \mathrm{~nm}$ (excited by $290 \mathrm{~nm}$ ); solid line, normal eyes; heavy line, nuclear cataracts. Right ordinate: percentage of insoluble protein (IP); dotted line, normal eyes; heavy dots, nuclear cataracts. Abscissa: age in years. The lines were drawn by hand. [Reproduced courtesy of S. Lerman and R. Borkman (Ref. 21) and Ophthalmic Res.]

occurs in the absence of any increase in insoluble (is) protein, the concentration of which starts rising only during the fifth decade. Similarly, nuclear cataracts contained in intact lenses (Figure 4) show a rise in fluorescence some 20 years prior to a rise in the concentration of is protein. ${ }^{21}$

There appears to be a real problem here since work on extracts from normal lenses, ${ }^{22}$ extended to brunescent cataracts, ${ }^{19}$ links an increase in fluorescence with a decrease in water-soluble protein. When Satoh, Bando, and Nakajima ${ }^{22}$ excited the (ui) moiety with $340 \mathrm{~nm}$ and measured at $420 \mathrm{~nm}$, fluorescence showed a steep rise after the age of 20 years, with that of the soluble material rising at only about $14 \%$ of that rate. There was also a systematic shift in the wavelength of maximum emission: $\gamma$-crystallin showed the shortest, with $\beta$-crystallin, $\alpha$-cystallin and (us) showing progressively longer maximal wavelengths.

Using high-pressure gel permeation chromatography, Bessems et al. ${ }^{23}$ were able to show that nontryptophan (nt) fluorescence increases with age by virtue of the age-related increase in the concentration of all the native crystallins with the exception of $\gamma_{2}$. They separated the cortex on the assumption that the inner $30 \%$ of the lens mass is the nucleus (Niesel, Kräuchi and Bachmann ${ }^{24}$ put the nuclear mass more nearly at $12 \%$ ). The presence of pigments was monitored in the supernatants following homogenization of the lenses by absorbance measurements at $280 \mathrm{~nm}$ (which would evidently miss 

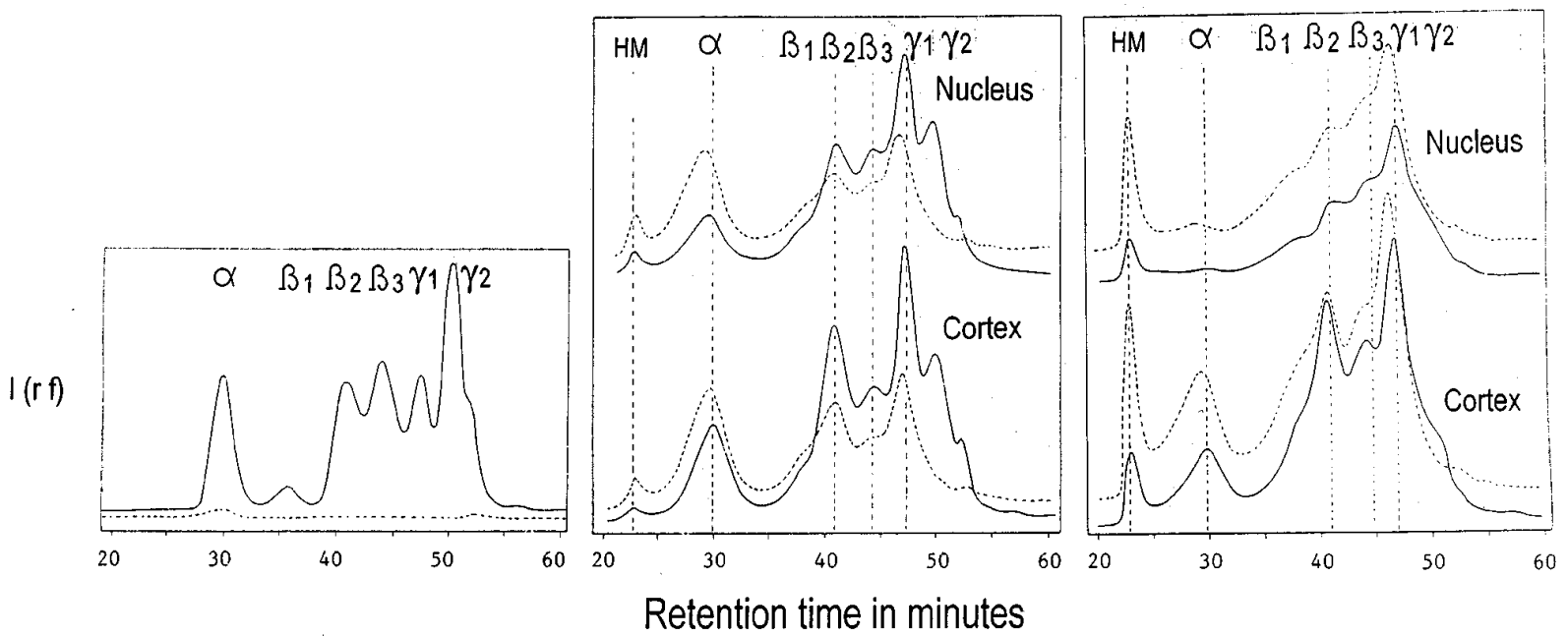

Fig. 5 Left: High-pressure gel permeation chromatography (HPGPC) of the water-soluble fraction from the lens of a newborn baby. Continuous line, absorbance measured at $280 \mathrm{~nm}$; broken line, relative fluorescence intensity excited with $360 \mathrm{~nm}$, and measured with $420 \mathrm{~nm}$. Center: HPGPC of the nuclear and cortical water-soluble fractions of a 29-year-old lens. Right: HPGPC of the nuclear and cortical water-soluble fractions for a clear 70-year-old lens. (After Ref. 23.)

pigments not showing any absorbance at this wavelength; cf. Ref. 25), and comparing this with the degree of fluorescence corresponding to calibrated molecular weights. Fluorescence was excited by 360 $\mathrm{nm}$ and measured at $420 \mathrm{~nm}$. Thus Fig. 5 (left to right) show traces for the two functions, with highmolecular-weight constitutents being on the left and low ones on the right for lenses of different ages. Note the swamping of $\gamma_{2}$ in Figure 5 (right), and the marked accumulation of high-molecularweight components after infancy.

Figure 6 summarizes the results for different crystallins on the left and shows that the age-related increase in nuclear fluorescence progresses at a faster rate than in the cortex. On the right, the dominant involvement of the nucleus is shown for nuclear cataracts: the onset of brunescence appears to be a watershed for an enhancement of fluorescence (see Ref. 21). The fluorescence of such lenses is partly attributable to the formation of 3,3'-bityrosine and anthranillic acid. ${ }^{25}$

The use of both intrinsic (i) and extrinsic (e) probes enabled Liang ${ }^{8}$ to study the microenvironment of fluorophors under observation; (i) included probes for both tryptophan (t) and nontryptophan (nt) fluorescence, while (e) included 4- $(N-$ iodoacetoxy) $\mathrm{N}$-methylamino-7-nitrobenz-2-oxa-1,3diazole (IANBD) and 6-(O-toluidinyl)naphthalene2-sulfonate (TNS). Measurements were obtained for emission maxima, relative quantum yields $\phi$ (which are proportional to the conversion efficiency $\mathrm{s}$, see below), and polarization P. The latter is a function of the ratio of parallel to perpendicular polarization of the emitted light, the exciting light being vertically polarized. The $(t)$ probe revealed constant values of $\phi$ and $P$ as a function of age, but decreases of the former, and increases in the latter

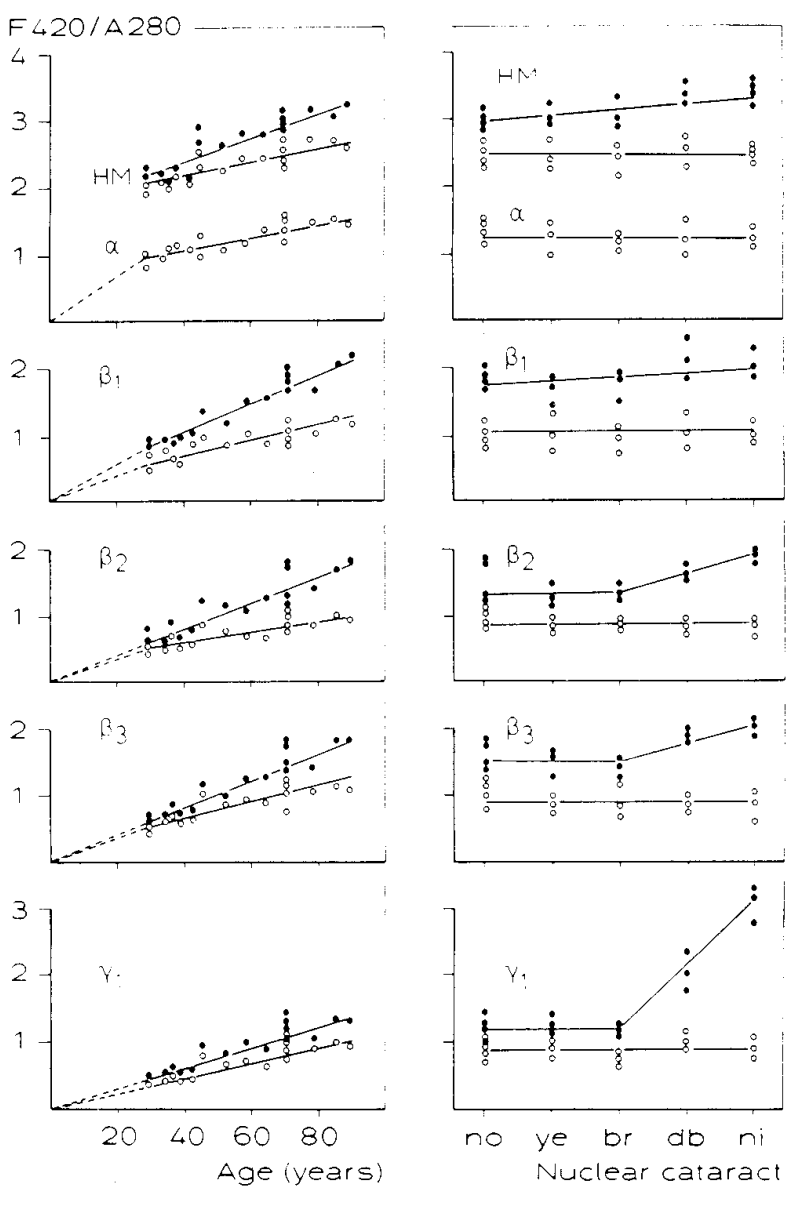

Fig. 6 Variation in the ratio of the relative fluorescence intensity measured at $420 \mathrm{~nm}$ to the absorbance at $280 \mathrm{~nm}$ for watersoluble protein in normal lenses (left) and nuclear cataracts (right). Empty circles, cortex; filled circles, nucleus. no, normal; ye, yellow; br, brown; db, dark brown; and ni, nigra. [Reproduced courtesy G. J. H. Bessems et al. (Ref. 23) and Invest. Ophthal. Vis. Sci.] 


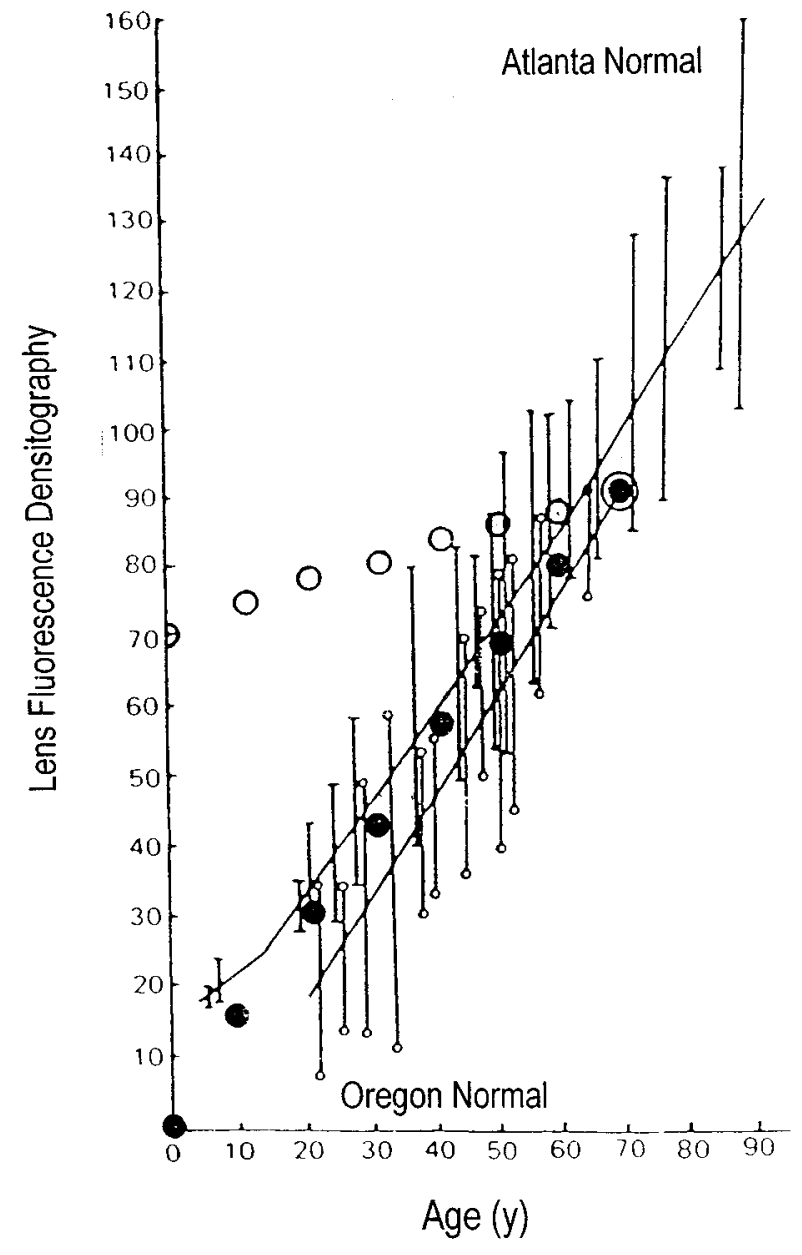

Fig. 7 Ordinate: lens fluorescence index, abscissa: age in years. The error bars ending in circles pertain to measurements in Oregon, the others to those in Atlanta. Filled circles, values predicted on the basis of absorbance and a variable conversion efficiency; empty circles, values predicted on the basis of absorbance and a constant conversion efficiency. (After Refs. 3 and 33.)

in glycated and high-molecular-weight $\alpha$-crystallin. In contrast, these compounds responded to (nt) with a rise in $\phi$ and a reduction in $P$. IANBD revealed a marked age-related rise in $\phi$.

Liang interprets this in terms of an unfolding of the parent molecule ${ }^{10}$ so that increasing numbers of fluorophor sites become exposed. By assuming that the conversion efficiency $s=1-\exp (-\beta A)$, where $A$ is the age in years and $\beta$ is the main term governing the increase in the age-related absorbance, ${ }^{26}$ good agreement can be obtained between theory and experiment as shown in Figures 1 and 7. ${ }^{3}$ The sparseness of Liang's age groups does not permit one to press the comparison, but, such as it is, the correlation between $\phi$ and $s$ is good (0.996). As predicted, ${ }^{27,28} s$ and $\phi$ increase with age.

This puts a complexion on the effects of lenticular senescence which has its attractions. The agerelated increase in fluorescence which Bleeker et al. ${ }^{29}$ attribute to a continued exposure to ambient short-wavelength radiation (see also Ref. 13) is not damaging itself, and, in the absence of any pathology, is not a sign of damage. But, as a mark of the molecule being unfolded, it is a harbinger of an increased vulnerability. And, that, after all, is one of the corollaries of senescence.

\section{IN VIVO STUDIES}

A number of informative studies on age-related variation in fluorescence have been published. For example, Scheimpflug biomicroscopy was used for living eyes between 17 and 85 years, although Hockwin and Lerman ${ }^{30}$ do not show a (linear) increase beyond 66 years. The traces produced by this method are less informative than those yielded by the methods listed below, but demonstrate nonetheless the presence of at least two fluorophores which were studied in more detail in vitro (see Sec. 4). Also, the peculiar transmissivity data (which changed systematically in the course of three publications spanning a decade) obtained from this laboratory, particularly for the ultraviolet part of the spectrum, raise some doubt about the radiometric reliability of this apparatus.

The spatially analytical quantification of fluorescence in the living human lens was introduced by Zeimer and Noth, ${ }^{31}$ and developed by subsequent workers. Commercially available fluorophotometers were used. The method is sketched in Figure 8. A short-waveband exciting beam enters the eye at a known distance from the cornea, and the resulting emitted beam is passed through a barrier filter that is nominally opaque to the exciting waveband. The intensity of the emergent beam is recorded. The distance between source and cornea is varied systematically so that a trace relating intensity to putative distance of penetration into the lens can be recorded. This is referred to as the lens profile (Figure 8 ). It is characterized by a marked proximal and a weak distal peak, the difference between which is barely noticeable when the ordinate is logarithmic. ${ }^{32}$ Measurements on thin lens slices ${ }^{4}$ suggest that this may be due to an artifact, possibly absorption: with one exception, the posterior peak was at all ages higher than the frontal one.

Zeimer and Noth ${ }^{31}$ stated that the ratio of the two amplitudes is equal to the transmissivity of the lens for the mean of the exciting and emitted wavebands. However, a spatial analysis of the fluorescence lens profile shows that, unless the exciting and emitted beams are collinear, the ratio is rather a measure of the transmissivity for the exciting radiation. ${ }^{3}$

The height of the first maximum is taken as a measure of fluorescence, and both Bleeker et al..$^{29}$ and Occhipinti, Mosier and Burnstein ${ }^{32}$ calibrated amplitude in terms of equivalents of fluorescein concentration. Its variation with age is shown in Figure 1. The black circles indicate theoretical values predicted on the basis of absorbance measure- 


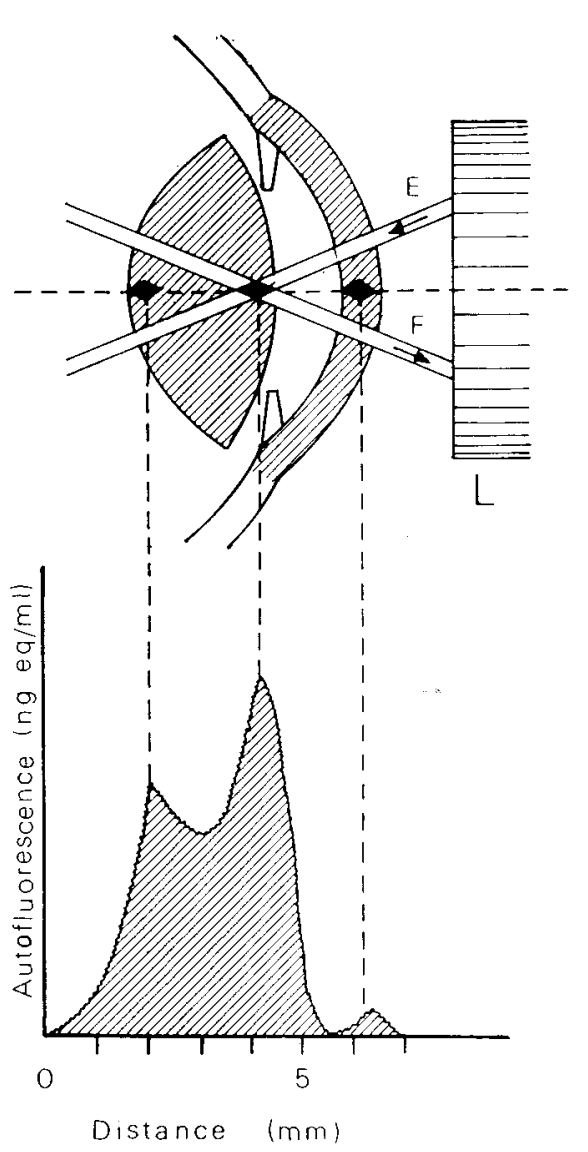

Fig. 8 In vivo fluorimetry. Top: $E$, exciting beam; $F$, measuring beam; and L, imaging system of fluorophotometer. Bottom: autofluorescence in nanogram equivalent of fluorescein per milliliter as a function of notional distance along the optic axis of the eye. [Courtesy J. C. Bleeker et al. (Ref. 29) and Invest. Ophthal. Vis. Sci.]

ments on excised lenses. ${ }^{3,26}$ Analogous results obtained for diabetic patients differ significantly (Figure 9): there is no simple model that would predict their data in terms of normal lens absorbance as in Fig. 1, and the reason is almost certainly that the diabetic pigment differs from the purely "aging" ones.

Lerman ${ }^{33}$ extended the procedure by defining the lens fluorescence index (LFI) in terms of the area of the lens profile rather than just in those of some of its features. He measured the LFI in two populations, namely, inhabitants of Atlanta and Oregon, respectively. The difference between the two (Figure 7) is attributed to the greater sunshine prevailing in Atlanta. The study is less valuable than it might be because it contains no information on whether diabetics were excluded.

Van Best et al. ${ }^{34}$ and Occhipinti, Mosier and Burnstein $^{32}$ followed Zeimer and Noth in the belief that the ratio of the amplitudes of the second to the first peak of the lens profile provides a measure of the transmissivity of the lens. While such values can evidently be computed, they tend to yield

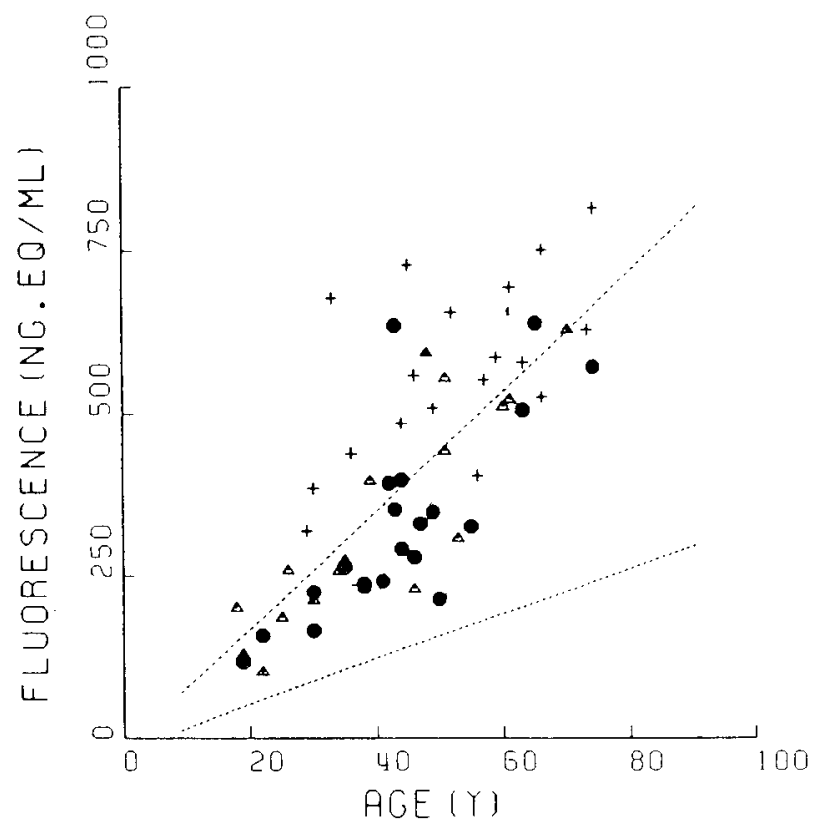

Fig. 9 Peak fluorescence (cf. Fig. 8) as a function of age of diabetic subjects. Dotted lines, $95 \%$ confidence limits for normal subjects (cf. Fig. 1); filled circles, duration of condition $<6 y$; triangles, 6 to $15 y ;+>15 y$. (After Ref. 29.)

transmissivities that are substantially higher than those determined by direct methods. Several reasons may be responsible for this. For example, the data may be contaminated by stray light, ${ }^{29}$ and the filters used may not cut off with the necessary efficiency. However, the relative ease with which data may be obtained makes the method very suitable for comparative measurements for aging lenses, and also for normal and pathological conditions.

\section{OBSERVATIONS IN VITRO}

The first detailed attempt at segregating the effects of absorbance on fluorescence measurements was undertaken by Jacobs and Krohn, ${ }^{4}$ who studied excised lenses under three conditions: (1) they illuminated the lens along the axis, and measured the fluorescence of the light emerging along an equatorial radius; (2) the lens was illuminated at $45 \mathrm{deg}$ to the axis, and the light emerging from the same point at 90 deg to this was measured, i.e., mainly cortical fluorescence was sampled; and (3) the lens was hemisected through the optic axis, and the arrangement of (2) applied to the central part of the nucleus.

It has to be said that the physical controls were unconventional. For example, the slit widths of the spectrophotofluorimeter were kept constant, which can have ensured neither quantal nor bandwidth constancy. For each lens, the wavelength of the exciting beam was varied "in order to determine the exciting wavelength at which the maximum fluorescence occurred." ${ }^{4}$ It was shown in Sec. 1.1 that 


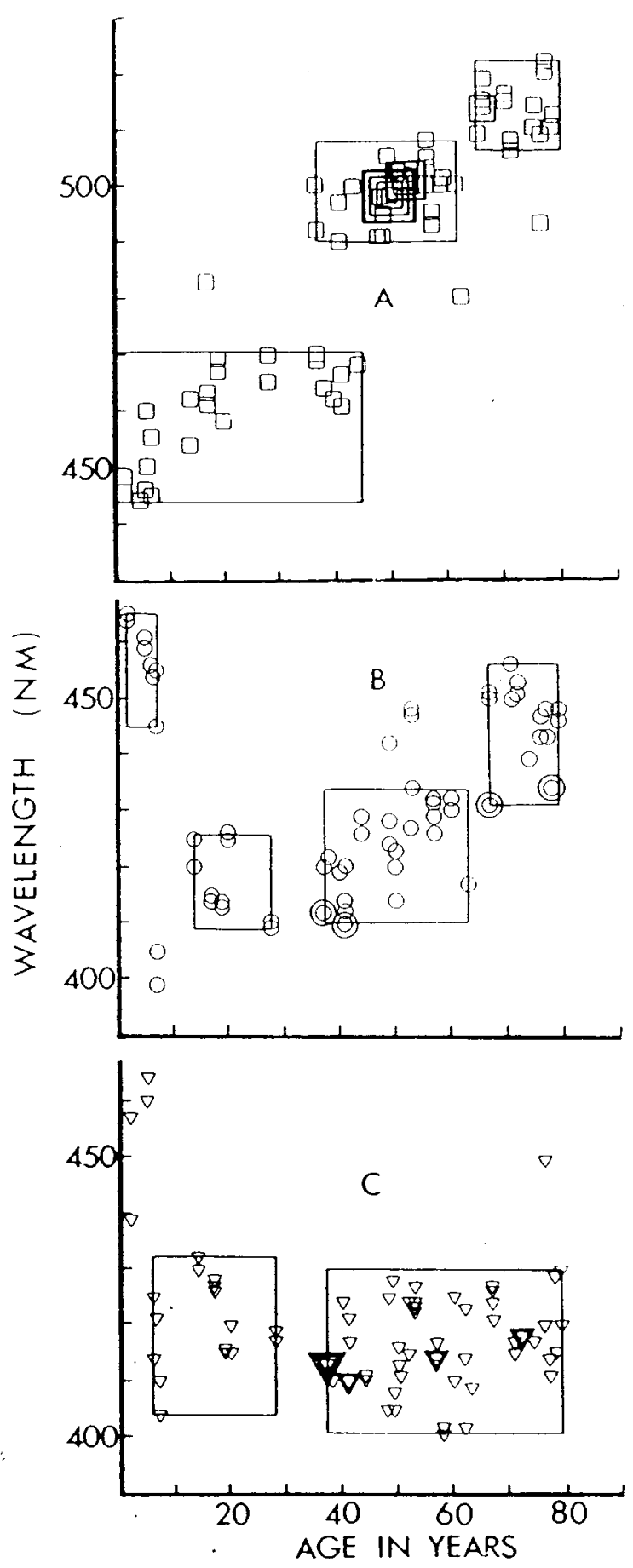

Fig. 10 (A) Wavelength of fluorescence maxima in whole human lenses as a function of age. The frames delineate clusters. Coincident values are indicated by concentric symbols. (B) The same data for the nucleus and (C) for the cortex. [Reproduced courtesy R. Jacobs and D. L. Krohn (Ref. 4) and J. Geront.]

this is unlikely to lead to unequivocal results. Nevertheless, Figure 10, which shows plots of the wavelengths of maximal fluorescence as a function of age for the above three conditions, emphasizes the need to distinguish cortical from nuclear portions at least regarding the type of fluorophor. It suggests
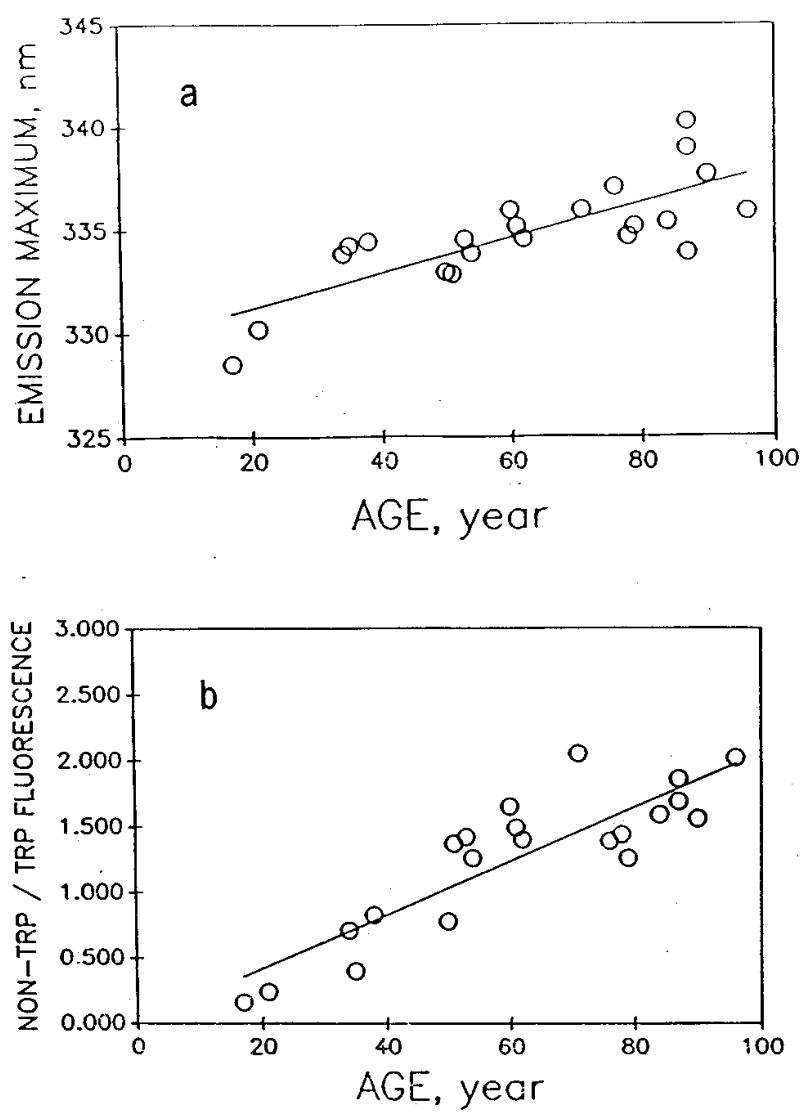

Fig. 11 Ratio of non-trp to trp fluorescence as a function of age; $p=0.0001$. (After Ref. 35.)

that the major part of the age-related change revealed in whole lenses is to be attributed to those occurring in the nucleus, and that the situation is relatively constant in the cortex. Jacobs and Krohn also give results for fluorescence intensity, but their manipulation of the data does not permit their results to be compared with others obtained later.

Though also adhering to apparently constant slit widths, Liang ${ }^{35}$ allowed for variations in spectral emittance and detector response, and also distinguished between fluorescence due to tryptophan (trp) and other substances (ntrp) respectively (see Sec. 4). The lenses were bisected through the equator and the flat (nuclear) surfaces studied. Figure 11(a) shows that the peak emission wavelength of (trp) fluorescence increases linearly with age, and its intensity predominates over (ntrp) only in young lenses (Fig. 11b).

It is difficult to assess the results obtained by Kurzel, Wolbarsht and Yamanash. ${ }^{7}$ Overlooking the fact that Cooper and Robson ${ }^{14}$ reported absorbance data on human lenses which "are extrapolations of measurements made on thin layers of lens material", and that the UV absorption maximum dropped in a sigmoid fashion, Kurzel and coworkers confined their attention to intact lenses in 

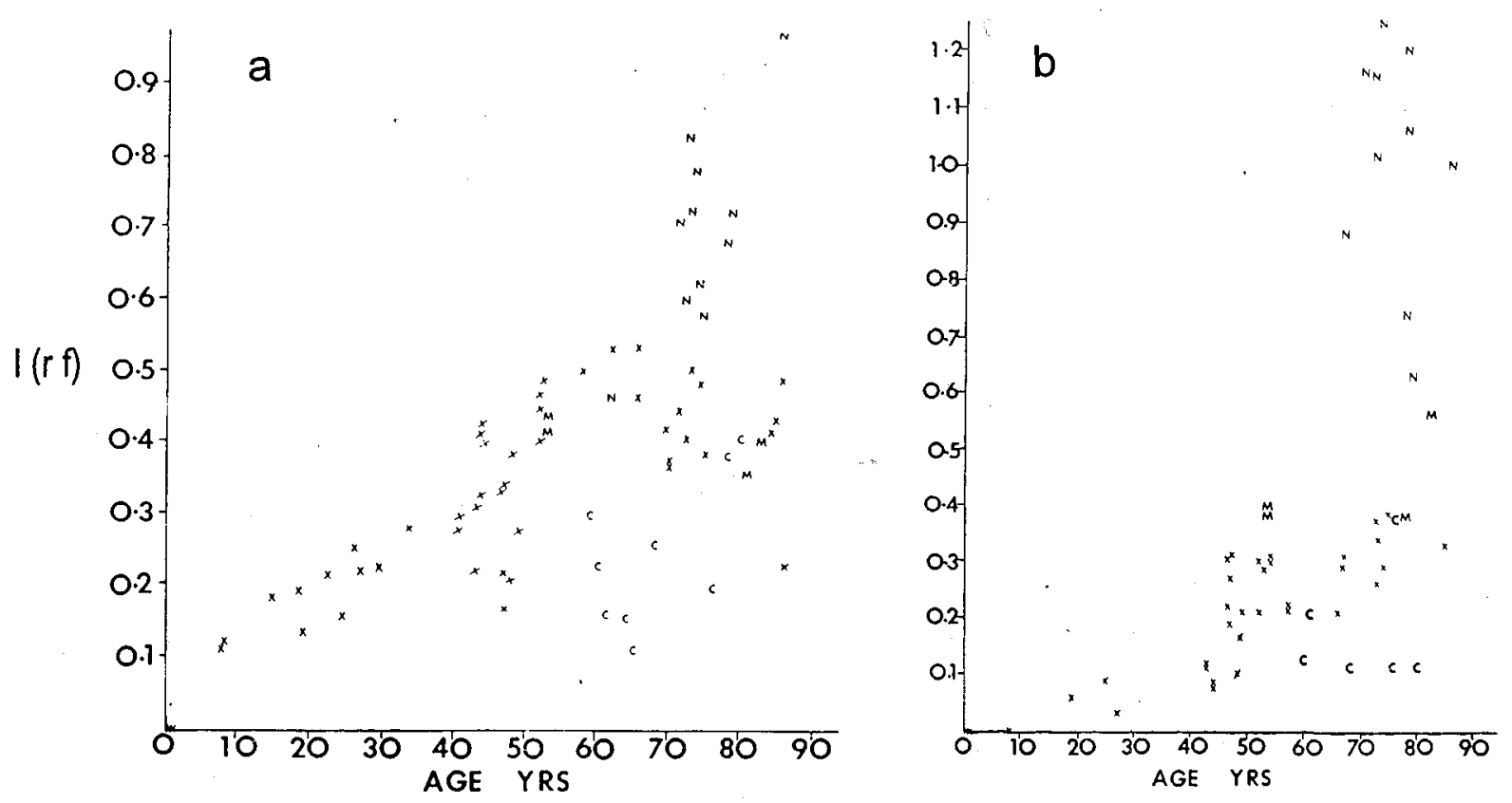

Fig. 12 Relative fluorescence $\mathrm{I}(\mathrm{r} f)$ in vivo as a function of age: (a) ratio of fluorescence intensity emitted at $440 \mathrm{~nm}$ (excited by $360 \mathrm{~nm}$ ) to fluorescence intensity emitted at $332 \mathrm{~nm}$ (excited by $295 \mathrm{~nm}$ ); and (b) ratio of fluorescence intensity emitted at 500 to $520 \mathrm{~nm}$ (excited by $435 \mathrm{~nm}$ ) to fluorescence intensity emitted at $332 \mathrm{~nm}$ (excited by $295 \mathrm{~nm}$ ). In both cases the denominator relates to tryptophan fluorescence (trp). $x$, normal; N, nuclear; C, cortical; $M$, mixed cataracts. (After Ref. 21. )

the age range of 39 to 81 years, and are unique in having "found no variation in the relative contributions of the spectral features with respect to age." It would, of course, have been interesting to learn what, if any, absolute changes occurred. No information is given on scaling constants relating to different emission wavelengths. Within the spectral range of 300 to $470 \mathrm{~nm}$, the authors claim to have detected the presence of 4 to 5 principal chromophores [fluorophors?]. However, fluorophors can be found at longer wavelengths, for example, at $480 \mathrm{~nm}$ and 500 to $510 \mathrm{~nm}$, depending on the exciting wavebands ${ }^{36}$ (see Ref. 13).

The effect of light scatter is liable to interfere with fluorescence measurements, a problem that is aggravated when fluorescence is measured in a spectral range which also shows much scatter. Lerman and Borkman ${ }^{13}$ attempted to surmount this difficulty, accentuated in the presence of cortical cataracts, by expressing their results as ratios of the fluorescence intensity measured in the visible part of the spectrum and that due to tryptophan fluorescence measured at $\sim 330 \mathrm{~nm}$. The rationale of the method is not explained; it is not immediately obvious how the experimental addition of two radiation intensities, due, for example, to a signal and noise, can be allowed for by a correction based on the ratio of intensities. It is also relevant to point out that there exists some confusion about Lerman and Borkman's work. They have corrected a reprint of Ref. 13 in my possession, which makes it clear that the graph appearing above the legend to Figure 2 is, in fact, Fig. 4; and vice versa (see Ref. 21). Further inked-in corrections relate to stimulus parameters.

That said, Lerman and Borkman found two fluorophors. One with an emission band in the bluegreen (500 to $520 \mathrm{~nm}$ ) accumulates faster after birth (Figure 12a) than does the other with an emission band at $440 \mathrm{~nm}$ (Fig. 12b). Since this spectral range can excite the fluorescence recorded in Figure 12a, ${ }^{36}$ there arises the question of whether secondary fluorescence may not perhaps have occurred and so contaminated the data: this has not been faced. Note that nuclear, brunescent cataracts present greatly accentuated fluorescence, but that cortical ones tend to be subnormal. This cannot come as a surprise if the absorption of light by a chromophore is a prerequisite for the occurrence of fluorescence: cortical cataracts represent discontinuities that have so far have not been associated with any pigmentation.

\section{LENTICULAR FLUORESCENCE AS NOISE}

It is remarkable how little attention the role of lenticular fluorescence in everyday vision has received. Dodt and Walther ${ }^{37}$ showed that electroretinographic responses of the cat are significantly affected by it, and that it is liable to vitiate the results of measurements. How does this happen? The stimulus to retinal responses has to be measured externally to the eye; hence an additional stimulus in the form of fluorescent, visually effective light 
sneaking into the system within the eye after the measurement has been made will facilitate a retinal response at a lower measured level than could be the case in its absence.

The question is one of magnitude and significance. It was shown in 1981, if not earlier, that the celebrated data on the age-related variation in human dark adaptation ${ }^{38}$ contain a paradox. When corrected for lenticular absorbance, they suggest that the oldest observers have a lower cone threshold than, say, middle-aged ones. Once fluorescence had been measured in an axially forward direction, and its visual equivalent had been computed from the data, ${ }^{36,39}$ Domey and McFarland's data for the highest age groups were found to be in error by an order of magnitude. This is of the same order as Van den Berg's ${ }^{9}$ more detailed data computed for a shorter wavelength range. Given the best available corrections for lenticular absorbance and fluorescence, the human visual threshold turns out to be remarkably constant over the major part of our lives. ${ }^{40}$

From an evolutionary point of view, it is noteworthy that the coloration of the lens assists in the reduction of chromatic aberration of the eye. ${ }^{41}$ However, the validity of this depends on a trade off between an illumination that optimizes visual acuity and minimizes untoward effects of lenticular fluorescence. A low level of fluorescence during the first half of our lives is helpful in that respect.

\section{REFERENCES}

1. A. Vogt, Lehrbuch und Atlas der Spaltlampenmikroskopie des lebenden Auges, Vol. II, Springer, Berlin (1931).

2. G. Klang, "Measurements and studies of the fluorescence of the human lens in vivo," Acta Ophthalmol. Kbh. Suppl. 31, p. 90 (1948).

3. R. A. Weale, "A theoretical link between lenticular absorbance and fluorescence," Proc. Roy. Soc. Lond. B (in press) (1996).

4. R. Jacobs and D. L. Krohn, "Variations in fluorescence characteristics of intact human crystalline lens segments as a function of age," J. Gerontol. 31, 641-647 (1976).

5. S. Lerman, O. Hockwin, and V. Dragomirescu, "In vivo lens fluorescence photography," Ophthalmic Res. 13, 224-228 (1981).

6. B. K. Pierscionek and D. Y. C. Chan, "The refractive index gradient of the human lens," Optom. Vis. Sci. 66, 822-829 (1989).

7. R. B. Kurzel, M. L. Wolbarsht, and B. S. Yamanashi, "Spectral studies on normal and cataractous intact human lenses," Exp. Eye Res. 17, 65-71 (1973).

8. J. N. Liang, "Fluorescence study of the effects of aging and diabetes mellitus on human lens alpha-crystallin," Curr. Eye Res. 6, 351-355 (1987).

9. T. J. T. P. Van den Berg, "Quantal and visual efficiency of fluorescence in the lens of the human eye," Invest. Ophthalmol. Vis. Sci. 34, 3566-3573 (1993).

10. J. J. Harding, "Conformational changes in human lens proteins in cataract," Biochem. J. 129, 97-100 (1972).

11. B. K. Pierscionek, "Refractive index of the human lens surface measured with an optic fibre sensor," Ophthalmic Res. 26, 32-36 (1993).

12. J. Dillon and S. J. Atherton, "Time resolved spectroscopic studies on the intact human lens," Photochem. Photobiol. 51, 465-468 (1990).

13. S. Lerman and R. Borkman, "Ultraviolet radiation in the aging and cataractous lens: a survey," Acta Ophthalmol. 56, 139-149 (1978).

14. G. F. Cooper and J. G. Robson, "The yellow colour of the lens of man and other primates," J. Physiol. 203, 411-417 (1969).

15. R. Van Heyningen, "The glucoside of 3-hydrokynurenine and other fluorescent compounds in the human lens," in The human lens-in relation to cataract, K. Elliott and D. W. Fitzsimons, Eds., pp. 151-168, Elsevier, London (1973).

16. D. Grover and S. Zigman, "Coloration of human lenses by near ultraviolet photo-oxydized tryptophan," Exp. Eye Res. 13, 70-76 (1972).

17. G. Cremer-Bartels, O. Hockwin, and C.-M. Schedtler, "The problem of fluorescence of human cataractous lenses," Ophthalmic Res. 3, 122-125 (1972).

18. S. Lerman, J. F. Kuck, Jr., R. F. Borkman, and E. Saker, "Induction, acceleration and prevention (in vitro) of an aging parameter in the ocular lens," Ophthalmic Res. 8, 213-226 (1976).

19. R. C. Augusteyn, "Distribution of fluorescence in the human cataractous lens," Ophthalmic Res. 7, 217-224 (1975).

20. M. Bando, Y. Ishii, and A. Nakajima, "Changes in blue fluorescence intensity and coloration of human lens protein with normal lens aging and nuclear cataract," Ophthalmic Res. 8, 456-463 (1976).

21. S. Lerman and R. Borkman, "Spectroscopic evaluation and classification of the normal, aging and cataractous lens," Ophthalmic Res. 8, 335-353 (1976).

22. K. Satoh, M. Bando, and A. Nakajima, "Fluorescence in human lens," Exp. Eye Res. 16, 167-172 (1973).

23. G. J. H. Bessems, E. Keizer, J. Wollensak, and H. J. Hoenders, "Non-tryptophan fluorescence of crystallins from normal and cataractous human lenses," Invest. Ophthalmol. Vis. Sci. 28, 1157-1163 (1987).

24. P. Niesel, H. Kräuchi, and E. Bachmann, "Der Abspaltungsstreifen in der Spaltlampenphotographie der alternden Linse," Graefe's Arch. Klin. Exp. Ophthalmol. 199, 11-20 (1976).

25. S. Garcia-Casteiras, J. Dillon, and A. Spector, "Nontryptophan fluorescence associated with human lens protein; apparent complexity and isolation of bityrosine and anthranilic acid," Exp. Eye Res. 26, 461-476 (1978).

26. R. A. Weale, "Age and the transmittance of the human crystalline lens," J. Physiol. 395, 577-587 (1988).

27. R. A. Weale, "Physical changes due to age and cataract," in Mechanisms of Cataract Formation in the Human Lens, G. Duncan, Ed., pp. 47-70, Academic Press, London (1981).

28. R. A. Weale, A Biography of the Eye-Development, Growth, Age, p. 267, H. K. Lewis, London (1982).

29. J. C. Bleeker, J. A. van Best, L. Vrij, E. A. van der Velde and J. A. Oosterhuis, "Autofluorescence of the lens in diabetic and healthy subjects by fluorophotometry," Invest. Ophthalmol. Vis. Sci. 27, 791-794 (1986).

30. O. Hockwin and S. Lerman, "Clinical evaluation of direct and photosensitized ultraviolet radiation damage to the lens," Ann. Ophthalmol. 14, 220-223 (1982).

31. R. C. Zeimer and J. M. Noth, "A new method of measuring in vivo the lens transmittance," and study of lens scatter, fluorescence and transmittance," Ophthalmic Res. 16, 246255 (1984).

32. J. R. Occhipinti, M. A. Mosier, and N. L. Burstein, "Autofluorescence and light transmission in the aging crystalline lens," Ophthalmologica 192, 203-209 (1986).

33. S. Lerman, "Human lens fluorescence aging index," Lens Res. 5, 23-31 (1988).

34. J. A. Van Best, E. W. S. J. Tjin A Tsoi, J. P. Boot, and J. A. Oosterhuis, "In vivo assessment of lens transmission for blue-green light by autofluorescence measurement," Ophthalmic Res. 17, 90-95 (1985).

35. J. N. Liang, "Front surface measurements of the age-related change in the human lens," Curr. Eye Res. 9, 399-405 (1990).

36. R. A. Weale, "Human lenticular fluorescence and transmissivity, and their effects on vision," Exp. Eye Res. 41, 457-473 (1985).

37. E. Dodt and J. B. Walther, "Fluorescence of the crystalline 
lens and electroretinographic sensitivity determinations," Nature 181, 286-287 (1958).

38. R. G. Domey and R. A. McFarland, "Dark adaptation as a function of age: individual prediction," Am. J. Ophthalmol. 51, 1262-1268 (1961)
39. R. A. Weale, "Retinal senescence," Retinal Res. 5, 53-73 (1986).

40. R. A. Weale, The Senescence of Human Vision, p. 175, Oxford University Press, Oxford (1992).

41. V. M. Reading and R. A. Weale, "Macular pigment and chromatic aberration," J. Opt. Soc. Am. 64, 231-234 (1974).

Acknowledgment: The Publishers wish to acknowledge permission received from publishers and authors to reproduce figures used in this paper. 